\title{
O crescimento de Aegla leptodactyla Buckup \& Rossi (Crustacea, Anomura, Aeglidae) ${ }^{1}$
}

\author{
Clarissa Köhler Noro ${ }^{2} \&$ Ludwig Buckup ${ }^{2}$
}

${ }^{1}$ Contribuição número 393 do Departamento de Zoologia, Instituto de Biociências, Universidade Federal do Rio grande do Sul.

2 Programa de Pós-Graduação em Biologia Animal, Departamento de Zoologia, Instituto de Biociências, Universidade Federal do Rio Grande do Sul. Avenida Bento Gonçalves 9500, prédio 43435, 91501-970 Porto Alegre, Rio Grande do Sul, Brasil.

\begin{abstract}
The growth of Aegla leptodactyla Buckup \& Rossi (Crustacea, Anomura, Aeglidae). The growth study, in length and weight, of Aegla leptodactyla Buckup \& Rossi, 1977, was developed based on biometric data of 2,435 specimens collected from Rio da Divisa, a tributary of the Rio Silveira, district of São José dos Ausentes, Rio Grande do Sul State, Brazil. The samplings were realized from April/2000 to July/2001. The captured specimens had recorded the Sex and the length and width of cephalothorax. The specimens from June/2000, November and May/2001 were weighted, and those from June and July/2001 had recorded their length and width of the chelipeds. For the description of the growth the von Bertalanffy's model was utilized. The growth curves in length $(\mathrm{mm})$, for data obtained through the modal progression, are described by the equations: $C_{t}=$ 19.83 [1- $\left.e^{-0,0023(t+55,5)}\right]$ for males and $C_{t}=18.096\left[1-e^{-0,0024(t+48,6)}\right]$ for females. For data obtained through the progression of age group means, the equations for males and females are, respectively, $C_{t}=18.15\left[1-e^{-0,0027(t+52)}\right]$ and $C_{t}=20.7$ [1- $e^{-0,002(t+63,4)}$ ]. For the growth description in weight $(\mathrm{g})$, for data obtained through modal progression, the equations are: $P_{t}=3.88\left[1-e^{-0,0023(t+55,5)}\right]^{3,073}$ for males and $P_{t}=2.78\left[1-e^{-0,0024(t+48,6)}\right]^{3,1046}$ for females, and for data obtained from progression of age group means: $P_{t}=2.96\left[1-e^{-0,0027(t+52)}\right]^{3,073}$ (males) e $P_{t}$ $=3.65\left[1-e^{-0,002(t+63,4)}\right]^{3,1046}$ (females). The analysis of covariance did not record significant differences between the length of right and left chelae, of males and females.
\end{abstract}

KEY WORDS. Crustacea, Aeglidae, Aegla leptodactyla, growth curve.

Crustáceos característicos das águas continentais da América do Sul, os anomuros do gênero Aegla Leach, 1820 são animais de hábitos bentônicos, encontrados em arroios e rios de correnteza, ocultos sob pedras e detritos vegetais. Destacam-se como elos importantes nas cadeias alimentares nos ambientes límnicos, pois, além de serem predadores de larvas aquáticas (Magni \& Py-Daniel 1989), constituem importante fonte alimentar para aves, rãs, jacarés e peixes (ArenAs 1976).

Na região neotropical, onde já foram descritas 61 espécies, o limite norte de distribuição do gênero Aegla é o Município de Franca, no Estado de São Paulo, Brasil, e ao sul ocorre até a Ilha de Madre de Diós, Província de Ultima Esperanza, Chile (BOND-Buckup \& Buckup 1994, JARA 1992, 1994).

Entre os estudos de caráter biológico e ecológico realizados com espécies de Aegla, destacam-se as contribuições de BaHAMONde \& López (1961) com Aegla laevis laevis (Latreille, 1818), López (1965) com Aegla paulensis Schmitt, 1942 (mencionada como A. odebrechtti paulensis) e Rodrigues \& Hebling (1978) com Aegla perobae Hebling \& Rodrigues, 1977. Em estudos mais recentes, BuENo \& Bond-Buckup (2000) trataram da dinâmica populacional de Aegla platensis Schmitt, 1942 e SwIECH-YAoub \& Masunari (2001b) estudaram a biologia reprodutiva de Aegla castro Schmitt, 1942.

Estudos biométricos foram realizados por VAZ-FERREIRA et al. (1945) com A. uruguayana Schmitt, 1942, por BAHAMONDE \& López (1961) com A. laevis laevis, por Rodrigues \& Hebling (1978) em A. perobae e por Swiech-Yaoub \& Masunari (2001a) com Aegla castro. A heteroquelia foi examinada em $A$. laevis laevis, $A$. paulensis, A. perobae e em A. platensis (BAHAMONDE \& LóPEZ 1961, López 1965, Rodrigues \& Hebling 1978, Bueno et al. 2000).

Dados sobre o crescimento das espécies de Aegla não são numerosos. O primeiro estudo que abordou o tema foi de Bahamonde \& López (1961), no Chile, com A. laevis laevis. O único trabalho sobre crescimento de Aegla utilizando o modelo de BertaLANFFy (1938) é de autoria de Bueno et al. (2000), em um estudo realizado com Aegla platensis no Arroio do Mineiro, na Bacia do Rio Gravataí, no Rio Grande do Sul.

Nota-se, dessa forma, que apesar do expressivo número 
de espécies descritas para a região neotropical, o estudo da dinâmica populacional, em especial do crescimento das espécies da família Aeglidae é pouco conhecido. Assim, tem-se por objetivo caracterizar o crescimento de uma espécie de ocorrência regional: Aegla leptodactyla Buckup \& Rossi, 1977. O estudo desta espécie reveste-se de um interesse especial pela circunstância de ela ocorrer em uma região de maior altitude onde temperaturas mais baixas da água, durante o inverno, podem determinar a existência de períodos temporalmente mais circunscritos de postura, com vantagem evidente para a identificação de grupos etários e suas modas e médias.

\section{MATERIAL E MÉTODOS}

As amostragens foram realizadas de abril de 2000 a julho de 2001, com periodicidade mensal, no curso superior do Rio da Divisa (28 38'17"S, 4957'56"W), no Município de São José dos Ausentes, Rio Grande do Sul. O Rio da Divisa é um afluente da margem esquerda do Rio Silveira, formador do Rio Pelotas e estende-se por uma área marginada pelos campos de cima da serra, com escassa vegetação marginal, numa altitude de aproximadamente $1.200 \mathrm{~m}$.

Os animais foram capturados com o auxílio de um puçá, numa extensão aproximada de $40 \mathrm{~m}$ lineares do rio, sendo este posicionado contra a correnteza de modo que, ao se revolver o substrato, os espécimens fossem carregados para dentro do equipamento de captura. O esforço de coleta foi variável, visando, a cada mês, a obtenção de número de animais suficiente para a caracterização dos grupos etários.

Para cada exemplar de Aegla leptodactyla foi registrado o sexo, pela observação de presença ou de ausência de pleópodos e poro genital na coxa do terceiro par de pereiópodos da fêmea. Foram registradas as medidas de comprimento do cefalotórax, desde a margem interna da órbita até o bordo posterior da carapaça e da maior largura do cefalotórax. Para tais medidas, foi utilizado um paquímetro digital (More \& Wright) com precisão de $0,01 \mathrm{~mm}$. Indivíduos menores que $6 \mathrm{~mm}$ de comprimento foram medidos em microscópio estereoscópico dotado de ocular micrométrica. Depois de feitas as medições, os animais foram devolvidos ao mesmo local de origem no Rio da Divisa.

Para a determinação da relação peso/comprimento, todos os animais medidos nos meses de junho/2000, novembro/2000 e maio/2001 foram também pesados. Para tal, utilizou-se uma balança digital (Fisatom) com precisão $0,01 \mathrm{~g}$. Na pesagem, foram desprezadas as fêmeas ovígeras e os indivíduos com ausência parcial de apêndices. Para verificar a hipótese de existência de heteroquelia, nos meses de junho e julho de 2001, os animais também tiveram registrado o comprimento e largura das quelas.

\section{Análise estatística}

O estudo do crescimento em comprimento de machos e fêmeas de Aegla leptodactyla, foi realizado pela análise das distribuições de freqüência por intervalo de classe de comprimento, ao longo dos meses de amostragem onde acompanhouse o comprimento médio do cefalotórax bem como as modas dos grupos etários. O uso das modas e das médias dos histogramas de freqüência, visou comparar a eficácia da escolha como base para a estimativa da forma de crescimento.

Para a análise do crescimento em comprimento foi utilizado o modelo de BERTALANFFY (1938) que corresponde à seguinte equação matemática: $C_{t}=C_{\infty}\left[1-e^{-k(t-t 0)}\right]$, onde: $\left(C_{t}\right)$ comprimento do cefalotórax na idade $\mathrm{t} ;\left(\mathrm{C}_{\infty}\right)$ comprimento médio máximo da carapaça; (e) base dos logaritmos naturais; (k) constante relacionada à taxa de crescimento; $\left(\mathrm{t}_{0}\right)$ período hipotético entre a fertilização e o nascimento (eclosão); (t) a idade considerada.

Para o ajuste das curvas de crescimento em comprimento do cefalotórax para machos e fêmeas foi utilizado o método de Ford-Walford (WALFord 1946) para intervalos de tempo desiguais entre amostras.

As curvas de crescimento obtidas para machos e fêmeas foram linearizadas através do método proposto por Allen (1976), e comparadas pela análise de covariância, para um intervalo de confiança $95 \%$, adotando-se os procedimentos recomendados por SNEDECOR \& COCHRAN (1967). Nesta análise foram realizados 3 testes: (1) a hipótese da homogeneidade das variâncias residuais; (2) a hipótese da igualdade entre a declividade ( $\beta$, do modelo linear) e (3) a hipótese de igualdade entre os intersectos (elevações, $\alpha$ do modelo linear).

A relação entre comprimento do cefalotórax e peso, para machos e fêmeas, foi descrita segundo RicKer (1975) pela equação: $\mathrm{P}=\mathrm{a} \cdot \mathrm{C}^{\mathrm{b}}$, onde: $(\mathrm{P})$ peso em gramas; $(\mathrm{C})$ comprimento do cefalotórax em milímetros; (a) fator de condição; (b) parâmetro da relação comprimento/peso.

O crescimento em peso foi calculado pela aposição do expoente (b) da curva comprimento/peso, ao modelo de Bertalanffy para o comprimento, como recomenda SANTOS (1978): $\mathrm{P}_{\mathrm{t}}=\mathrm{P}_{\infty}\left[1-\mathrm{e}^{-\mathrm{k}(\mathrm{t}-\mathrm{t} 0}\right]^{\mathrm{b}}$, onde: $\left(\mathrm{P}_{\mathrm{t}}\right)$ peso total médio dos indivíduos com idade $t ;\left(\mathrm{P}_{\infty}\right)$ peso médio máximo que os indivíduos podem atingir; (k) parâmetro relacionado com a taxa de crescimento, referente a dias; (e) base dos logaritmos naturais; $\left(\mathrm{t}_{0}\right)$ período hipotético entre a fertilização e o nascimento (eclosão); (b) parâmetro relacionado com o comprimento e peso.

Para verificar a possível ocorrência de crescimento diferencial dos quelípodos, entre os sexos, foram estimadas regressões do comprimento da quela direita para a quela esquerda, para machos e fêmeas. As retas foram comparadas pela análise de covariância para intervalo de confiança de $95 \%$ e $99 \%$, adotando-se os procedimentos recomendados por SNEDECOR \& Cochran (1967).

\section{RESULTADOS E DISCUSSÃO}

Ao longo do período amostral, foram obtidos dados de comprimento e largura cefalotorácica de 2.435 exemplares de Aegla leptodactyla, sendo 1.323 machos e 1.112 fêmeas. O menor exemplar capturado foi um juvenil com 2,15 mm de comprimento de cefalotórax e animais com comprimento menor de $6 \mathrm{~mm}$, foram considerados numericamente para ambos os sexos. BAHAMONDE \& Lopéz (1961) registraram a presença de pleópodos apenas em fêmeas maiores de $12 \mathrm{~mm}$ de comprimento de carapaça, não apresentando dados para o aparecimento do poro genital, porém, Swiech-Ayoub \& Masunari (2001b) observaram que algumas fêmeas com $6,5 \mathrm{~mm}$ de comprimento de carapaça já apresentavam tal dimorfismo sexual secundário. A comparação dos dados de comprimento de cefalotoráx obtidos na presente investigação com os resultados apresentados por outros autores, como BAHAMONDE \& LÓPEZ (1961), López (1965), Rodrigues \& Hebling (1978) e Swiech-Ayoub \& Masunari $(2001 \mathrm{a}, \mathrm{b})$ fica prejudicada pela circunstância de estes terem sido obtidos com a inclusão do rostro. Na presente con- 

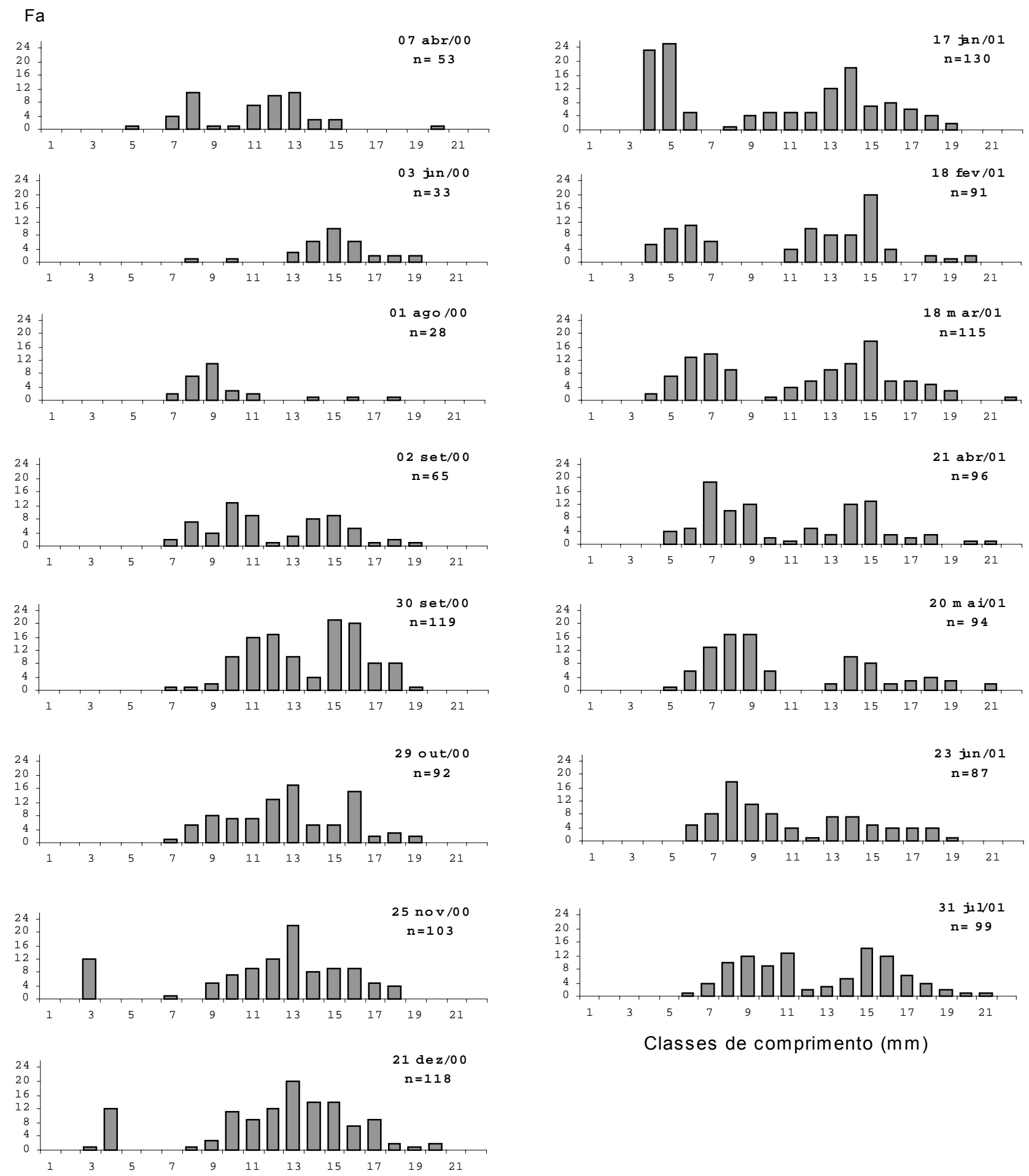

Figura 1. Distribuição das freqüências absolutas mensais do comprimento do cefalotórax $(\mathrm{mm})$ de machos de Aegla leptodactyla coletadas de abril/2000 a julho/2001 no Rio da Divisa, São José dos Ausentes, Rio Grande do Sul. Fa: freqüência absoluta; Classes de comprimento

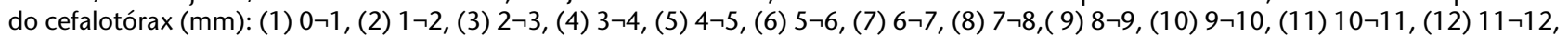

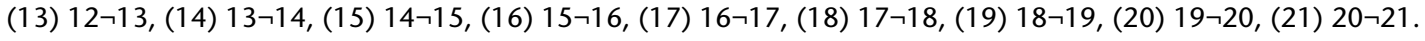



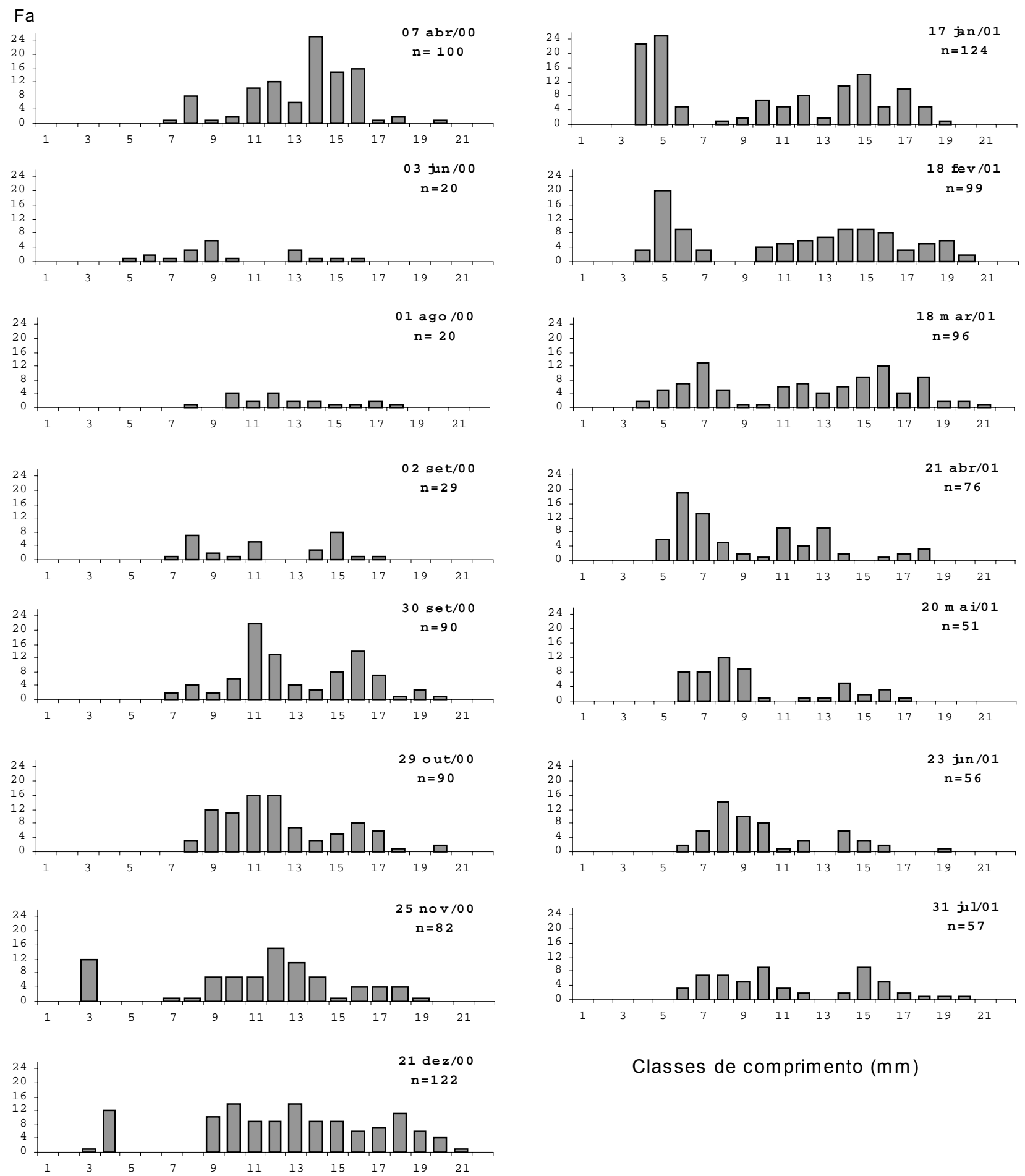

Classes de comprimento $(\mathrm{mm})$

Figura 2. Distribuição das freqüências absolutas mensais do comprimento do cefalotórax ( $\mathrm{mm}$ ) de fêmeas de Aegla leptodactyla coletadas de abril/2000 a julho/2001 no Rio da Divisa, São José dos Ausentes, Rio Grande do Sul. Fa: freqüência absoluta; Classes de comprimento

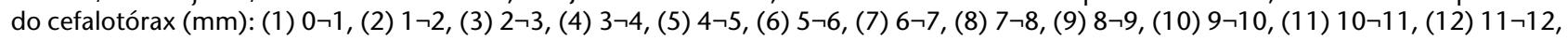

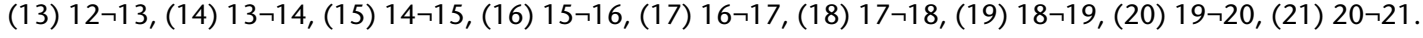

Revista Brasileira de Zoologia 20 (2): 191-198, junho 2003 
tribuição a medida do comprimento do cefalotoráx foi obtida a partir da margem da órbita, já que o rostro revela-se, com freqüência, com seu ápice truncado.

Para a determinação do tipo de crescimento relativo entre o comprimento e largura do cefalotórax, foram obtidas as seguintes equações de regressão: $\mathrm{Y}=0,906 \mathrm{x}-0,1528(\mathrm{r}=0,99)$ para machos e $\mathrm{Y}=0,9492 \mathrm{x}-0,4432(\mathrm{r}=0,99)$ para fêmeas. As retas indicam forte correlação entre os dois parâmetros, demonstrando um crescimento isométrico das medidas de largura e comprimento do cefalotórax.

As distribuições de freqüências absolutas mensais de comprimento do cefalotórax em milímetros para machos e fêmeas estão ilustradas nas figuras 1 e 2 , respectivamente. Os histogramas de freqüência classes de comprimento de $A$. leptodactyla mostram modas bastante nítidas para os grupos etários ao longo dos meses de amostragem. Este fato poderia ser atribuído à circunstância da espécie ser endêmica em uma região de altitude elevada, onde as temperaturas são relativamente mais baixas, determinando períodos de postura mais circunscritos. Nota-se, a partir do mês de novembro, a presença de três grupos etários, que representam uma geração de animais juvenis que entraram na população (recrutamento), uma geração de animais adultos e uma terceira geração, com pequena freqüência de indivíduos mais velhos que vão desaparecendo nos meses subseqüentes. Durante as amostragens foi possível observar apenas um período de recrutamento ao ano, como observado em A. laevis laevis, A. paulensis, A. perobae e A. platensis (BAHAMonde \& López 1961, López 1965, Rodrigues \& Hebling 1978, Bueno \& Bond-Buckup 2000). Já em A. castro, Swiech-Ayoub \& MASUNARI (2001b), verificaram dois pulsos de recrutamento ao ano, em abril e dezembro, embora tenham registrado juvenis para todo o período amostral, o que dificulta a identificação dos grupos etários com vistas a estimação das modas.

Nos machos, durante os 15 meses de amostragem, a classe de comprimento com maior freqüência absoluta foi de $15 \mathrm{~mm}$ e nas fêmeas, a classe 11 foi a que obteve a freqüência absoluta máxima. Em ambos os sexos, observa-se uma diminuição acentuada das freqüências a partir da classe $17 \mathrm{~mm}$, o que pode ser resultado da mortalidade prevista nas classes de comprimento mais antigas. Apesar das observações de López (1965) sobre o deslocamento de indivíduos de Aegla odebrchtti paulensis por uma distância de até $300 \mathrm{~m}$ ao longo de um arroio na reserva Florestal do Alto da Serra em São Paulo, não há dados suficientes para a emissão de hipóteses sobre um possível deslocamento de animais mais velhos para outros ambientes que escapem aos procedimentos de amostragem.

Animais de dimensões menores, expressando o recrutamento, somente foram coletados a partir de novembro de 2000. A inclusão destes dados no estudo torna-se essencial para o cálculo dos valores da curva de crescimento. Assim, partindose da premissa de que a forma de crescimento das populações locais não sofre mudanças importantes de um ano para outro, decidiu-se incorporar ao estudo do deslocamento modal e das médias o coorte de jovens amostrados a partir de novembro de 2000, antepondo-as ao grupo etário representado pelos animais maiores, amostrados nos meses de abril de 2000 a outubro de 2000. A tabela I informa sobre o processo de intercalação de valores aqui descrito.

As curvas de crescimento em comprimento, para machos e fêmeas, calculadas através do modelo de BERTALANFFy (1938) foram, para dados obtidos através do deslocamento modal (Figs
Tabela I. Modas e médias utilizadas para o cálculo das curvas de crescimento de machos e fêmeas de Aegla leptodactyla.

\begin{tabular}{|c|c|c|c|c|c|c|c|}
\hline \multicolumn{4}{|c|}{ Machos } & \multicolumn{4}{|c|}{ Fêmeas } \\
\hline Dia & Modas & Dia & Médias & Dia & Modas & Dia & Médias \\
\hline $25 \mathrm{nov} / 00$ & 2,50 & $25 \mathrm{nov} / 00$ & 2,50 & $25 \mathrm{nov} / 00$ & 2,50 & $25 \mathrm{nov} / 00$ & 2,5 \\
\hline $25 \mathrm{mai} / 01$ & 8,00 & $07 \mathrm{abr} / 00$ & 7,30 & $18 \mathrm{mar} / 01$ & 6,40 & $18 \mathrm{mar} / 01$ & 6,1 \\
\hline $30 \mathrm{set} / 00$ & 11,12 & $30 \mathrm{set} / 00$ & 10,78 & $31 \mathrm{jul} / 01$ & 9,40 & 03 jun/00 & 8,0 \\
\hline $25 \mathrm{nov} / 00$ & 12,14 & $21 \mathrm{dez} / 00$ & 12,80 & 02 set/00 & 10,40 & 30 set/00 & 10,5 \\
\hline $17 \mathrm{jan} / 01$ & 13,30 & $18 \mathrm{mar} / 01$ & 14,16 & $21 \mathrm{dez} / 00$ & 12,05 & $18 \mathrm{fev} / 01$ & 13,1 \\
\hline $18 \mathrm{mar} / 01$ & 14,36 & $31 \mathrm{jul} / 01$ & 14,58 & $18 \mathrm{fev} / 01$ & 13,00 & $31 \mathrm{jul} / 01$ & 15,2 \\
\hline \multirow[t]{2}{*}{31 jul/01 } & 14,81 & & & $20 \mathrm{mai} / 01$ & 13,57 & & \\
\hline & & & & 31 jul/01 & 14,63 & & \\
\hline
\end{tabular}

3 e 4): $C_{t}=19,83\left[1-\mathrm{e}^{-0,0023(t+55,5)}\right]$ (machos) e $C_{t}=18,96\left[1-\mathrm{e}^{-0,0024}\right.$ (t+48,6)] (fêmeas) e para dados obtidos através do deslocamento das médias (Figs 5 e 6 ): $C_{t}=18,15\left[1-\mathrm{e}^{-0,0027(t+52)}\right]$ (machos) e $\mathrm{C}_{\mathrm{t}}=20,7\left[1-\mathrm{e}^{-0,002(\mathrm{t}+63,4)}\right]$ (fêmeas).

$\mathrm{O}$ valor de $\mathrm{t}_{0}$ foi calculado pelo método de Fabens (1965) obtendo-se (com arredondamento para valores inteiros) $-55,5$ dias para machos, e $-48,6$ dias para fêmeas (pela análise do deslocamento modal), e $-52,0$ dias para machos e $-63,4$ dias para fêmeas (pela análise do deslocamento das médias).

Os comprimentos médios máximos do cefalotórax, bem como a taxa de crescimento, estimados pela curva de crescimento em comprimento, através da análise do deslocamento modal e pela análise do deslocamento das médias, foram semelhantes entre os sexos. Porém, pelos dados obtidos, verificou-se que em ambas as análises, com exceção da análise do deslocamento das médias de fêmeas, os valores do comprimento assintótico ficaram subestimados quando comparados com dados obtidos no campo.

Para Aegla platensis, BuENo et al. (2000) encontraram as seguintes curvas de crescimento, obtidas através do deslocamento das modas: $C_{t}=17,39\left[1-e^{-0,0041(t+39,13)}\right]$ para machos e $C_{t}=19,12\left[1-e^{-0,0033(t+50,38)}\right]$ para fêmeas. Comparando-se estes resultados com os obtidos no presente estudo, observa-se que os tamanhos médios máximos encontrados para machos são inferiores aos valores encontrados para $A$. leptodactyla enquanto que os tamanhos médios máximos das fêmeas são muito semelhantes. Também foi constatada diferença na taxa de crescimento das duas espécies, sendo que os valores de $\mathrm{k}$ encontrados para machos e para fêmeas de A. leptodactyla apresentaram valores inferiores daqueles de $A$. platensis.

$\mathrm{Na}$ tabela II estão apresentados os resultados da comparação das curvas de crescimento linearizadas, onde é possível constatar, pela alternativa da análise de progressão das modas, de machos e fêmeas, que não existem diferenças significativas na homogeneidade das variâncias residuais pois $\mathrm{F}_{c}=0,05<$ $\mathrm{F}_{0,05, \mathrm{gl} 4,4}$ ao contrário de BuENo et al. (2000) que rejeitam tal hipótese ao estudar o crescimento de Aegla platensis. Os mesmos autores não encontraram diferenças significativas na comparação entre declividade e entre elevação das retas, o que não ocorre neste estudo, sendo que entre as declividades $F_{c}=114,7$ $>\mathrm{F}_{0,05, \mathrm{gl} 1,8}$ e entre as elevações $\mathrm{F}_{\mathrm{c}}=108,3>\mathrm{F}_{0,05, \mathrm{gl} 1,9}$ onde podemos 

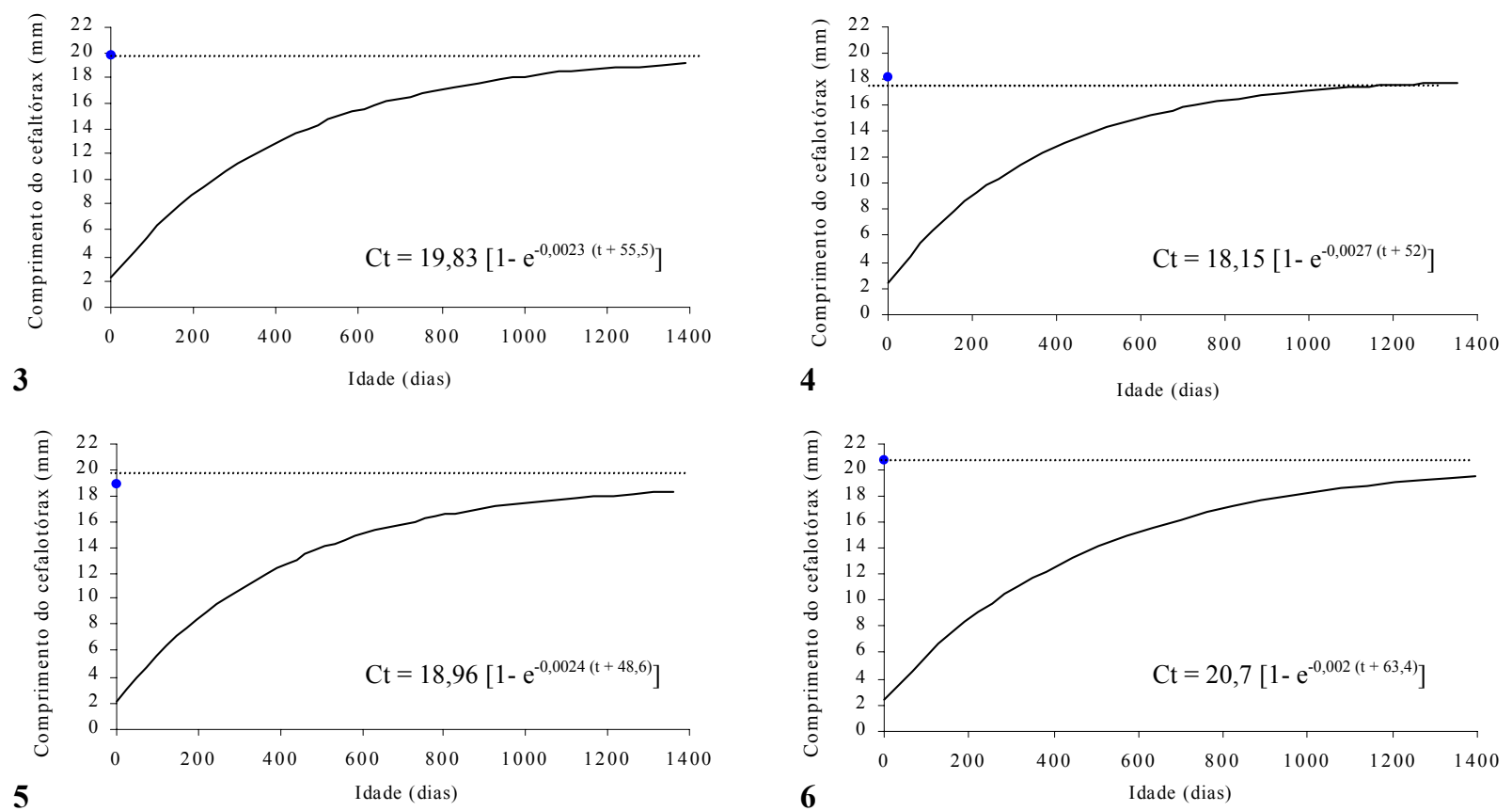

Figuras 3-6. Curva de crescimento em comprimento do cefalotórax de exemplares de Aegla leptodactyla coletados no período de abril/ 2000 a julho/2001, no Rio da Divisa, São José dos Ausentes, Rio Grande do Sul. Machos, (3) pela análise do deslocamento de modas; (4) pela análise do deslocamento de médias. Fêmeas, (5) pela análise do deslocamento de modas; (6) pela análise do deslocamento de médias. (Ct) representa o comprimento do cefalotórax $(\mathrm{mm})$ dos indivíduos no tempo t; (Ca) representa o comprimento médio máximo e (t) é a idade em dias.

Tabela II. Comparação entre as regressões do comprimento $(\mathrm{mm})$ para a idade (dias), para machos e fêmeas, pela análise do deslocamento das modas e pela análise do deslocamento das médias.

\begin{tabular}{lccc}
\hline \multicolumn{1}{c}{ Sexo } & $\mathrm{F}\left(\mathrm{s}^{2}\right)$ & $\mathrm{F}(\mathrm{a})$ & $\mathrm{F}(\mathrm{b})$ \\
\hline Macho/fêmea (Modas) & $\mathrm{F}(0,05) \mathrm{NS}$ & $\mathrm{F}(108,3)^{\star}$ & $\mathrm{F}(114,7)^{\star}$ \\
Macho/fêmea (Médias) & $\mathrm{F}(0,02) \mathrm{NS}$ & $\mathrm{F}(2,2) \mathrm{NS}$ & $\mathrm{F}(40,8)^{\star}$ \\
Machos (modas)/machos (médias) & $\mathrm{F}(0,04) \mathrm{NS}$ & $\mathrm{F}(7,0)^{\star}$ & $\mathrm{F}(42,0)^{\star}$ \\
Fêmeas (modas)/ fêmeas (médias) & $\mathrm{F}(0,02) \mathrm{NS}$ & $\mathrm{F}(10,8) \mathrm{NS}$ & $\mathrm{F}(22,3)^{\star}$ \\
\hline
\end{tabular}

$F\left(s^{2}\right)$ valor de $F$, calculado pela comparação das variâncias residuais; $F(a)$ valor de $F$, calculado pela comparação dos valores de elevações; $F(b)$ valor de $F$ calculado pela comparação dos valores de declividade; (*) significante para $\alpha \leq$ 0,05 ; (NS) não significante para $\alpha=0,05$.

concluir que existe diferença significativa entre os valores de $b$ e de a de machos e fêmeas. Pode-se concluir, nas demais comparações de retas, que não houve diferenças significativas entre as variâncias residuais, o que confere validade à análise de covariância realizada. A hipótese de igualdade entre declividades foi rejeitada em todas as comparações realizadas. No teste de igualdade entre as elevações foi verificado que não existem diferenças significativas entre as retas de machos e fêmeas pela análise da progressão das médias, onde $\mathrm{Fc}=2,20<\mathrm{F}_{0,05, \mathrm{gl}}$ 1,9' sendo que, nas demais retas comparadas, constatou-se diferen- ças significativas entre os valores de a. Estes dados indicam que a utilização de modas bem como de médias mostram um crescimento diferenciado entre os sexos, o que garante tratar o crescimento de machos e fêmeas com curvas separadas.

Hartnoll (1985) menciona que o crescimento em anomuros, apesar das limitadas informações, pode ser considerado como indeterminado, ou seja, o animal sofre ecdises contínuas após a puberdade. Isso, porém, não significa um crescimento ilimitado, pois como podemos observar na curva de crescimento de A. leptodactyla, bem como para os demais crustáceos, o crescimento se torna progressivamente mais lento com o aumento do tamanho do animal.

Segundo Hartnoll (1982) e Passano (1960), o crescimento dos crustáceos geralmente é similar entre os sexos até a maturidade. A partir deste período, porém, há uma diferença aparente, com períodos de intermuda mais longos nas fêmeas, freqüentemente associada à incubação dos ovos. Este fato pode ser constatado nas amostragens de campo, onde o comprimento do maior macho $(21,07 \mathrm{~mm})$ foi superior ao da maior fêmea $(20,59 \mathrm{~mm})$, fato também observado por BAHAMONDE \& López (1961) em Aegla laevis laevis; López (1965) em Aegla odebrechtti paulensis; Rodrigues \& Hebling (1978) em Aegla perobae; Bueno et al. (2000) em Aegla platensis e Swiech-Ayoub $\&$ Masunari (2000a) em Aegla castro.

A longevidade de $A$. platensis foi estimada em de 2,5 anos (Bueno \& Bond-Buckup 2000) e de A. castro, de dois anos (SwiechAyoub \& Masunari 2001b). BaHAMonde \& Lopéz (1961) estimaram

Revista Brasileira de Zoologia 20 (2): 191-198, junho 2003 


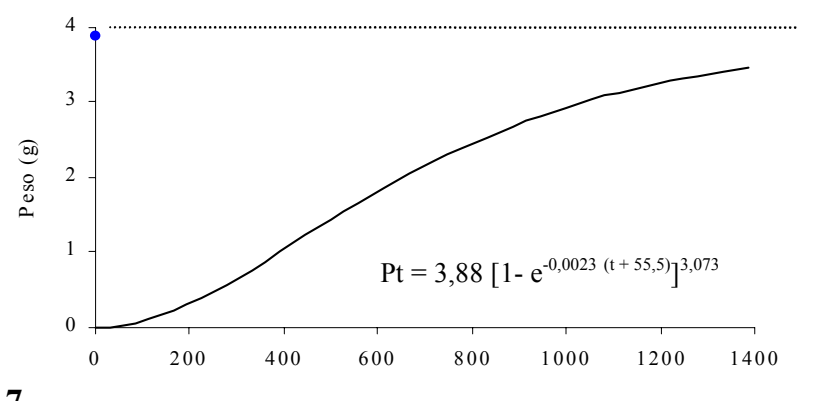

7

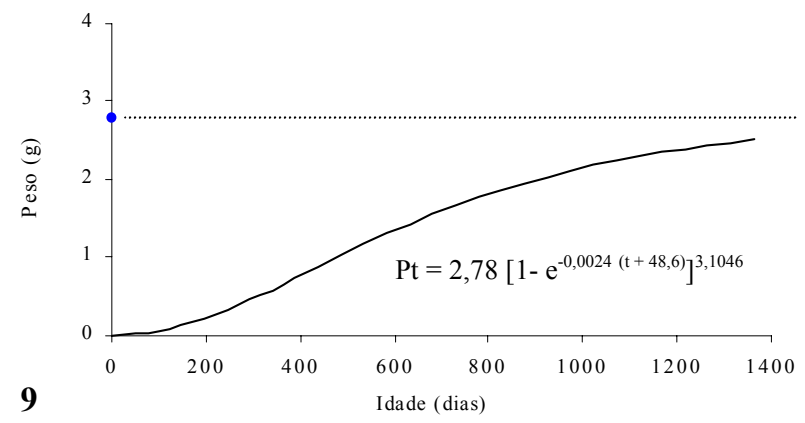

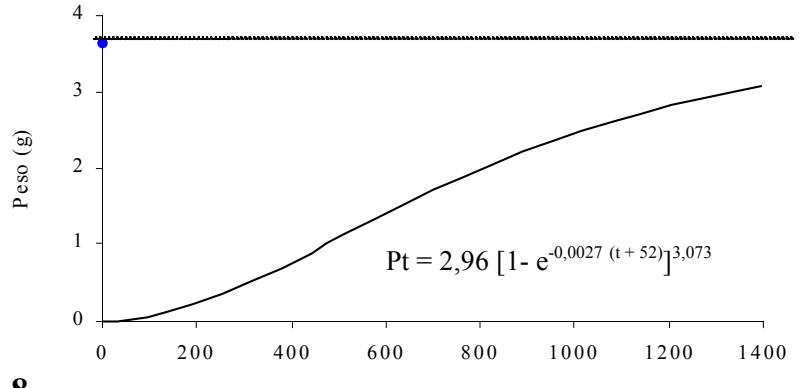

8

Idade (dias)

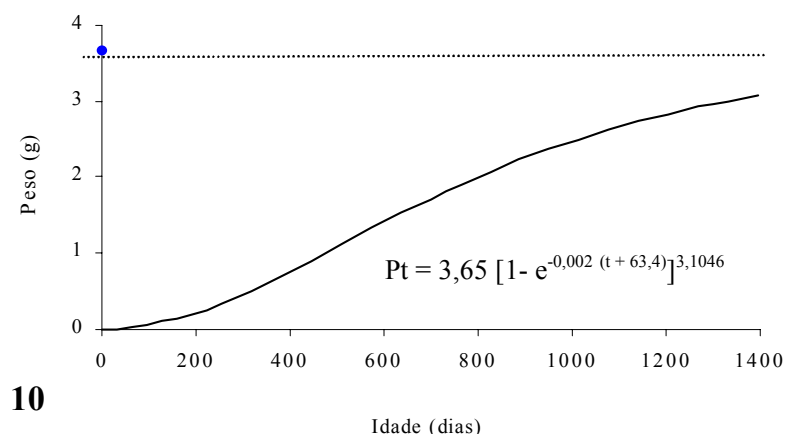

Figuras 7-10. Curva de crescimento em peso de exemplares de Aegla leptodactyla coletados no período de abril/2000 a julho/2001, no Rio da Divisa, São José dos Ausentes, Rio Grande do Sul. Machos, (7) pela análise do deslocamento de modas; (8) pela análise do deslocamento de médias. Fêmeas, (9) pela análise do deslocamento de modas; (10) pela análise do deslocamento de médias. (Pt) representa o peso $(\mathrm{g})$ dos indivíduos no tempo $\mathrm{t}$; $(\mathrm{Pa})$ representa o peso médio máximo e ( $\mathrm{t}$ ) é a idade em dias.

a longevidade de A. laevis laevis, pelo método de Petersen, em 37 meses. Considerando-se que o valor assintótico de comprimento de A. leptodactyla ficou subestimado no presente estudo, entende-se que uma estimativa da longevidade baseada na curva de crescimento ficaria prejudicada. Assim, restaria emitir a hipótese de que aos dois anos e meio de idade, os machos de $A$ leptodactyla poderiam atingir no mínimo, 17,69 mm de comprimento cefalotorácico.

A relação entre o peso e o comprimento do cefalotórax foi estimada com dados de 171 machos e 109 fêmeas de Aegla leptodactyla coletadas nos meses de junho/2000, novembro/ 2000 e maio/2001, e são descritas pelas equações: $P_{t}=0,0004$.C $3,073(r=0,99)$ para machos e $P_{t}=0,0003 . C^{3,1046}(r=0,99)$ para fêmeas. As equações descreveram satisfatoriamente a relação entre peso e comprimento de A. leptodactyla, o que pode ser evidenciado pelos elevados valores do coeficiente de correlação. Segundo Hartnoll (1982), o valor da constante b está relacionada com o tipo de crescimento apresentado pelo indivíduo, sendo o crescimento isométrico, como o registrado para a espécie aqui estudada, o peso é proporcional ao cubo do comprimento. Relações deste tipo também foram registradas por BUENO et al. (2000) para A. platensis, sendo a relação peso/comprimento de machos e fêmeas descrita pelas equações $P_{t}=0,000421$.C ${ }^{3,0427}$ e $\mathrm{P}_{\mathrm{t}}=0,000417 . \mathrm{C}^{3,0546}$.

As equações que descrevem o crescimento em peso, com dados obtidos a partir de progressão das modas são (Figs 7 e 8):
$P_{t}=3,88\left[1-e^{-0,0023(t+55,5)}\right]^{3,073}$ (machos) e $P_{t}=2,78\left[1-e^{-0,0024(t}\right.$ $\left.{ }^{+48,6)}\right]^{3,1046}$ (fêmeas). Para dados obtidos a partir de progressão das médias (Figs 9 e 10): $P_{t}=2,96\left[1-e^{-0,0027(t+52)}\right]^{3,073}$ (machos) e $P_{t}=3,54\left[1-e^{-0,002(t+63,4)}\right]^{3,1046}$ (fêmeas).

Através das curvas de crescimento em peso verifica-se que os machos de $A$. leptodactyla atingem pesos médios superiores aos das fêmeas, pela análise de modas. Pela análise de médias os machos atingem pesos médios inferiores, devido ao fato de o comprimento médio máximo do cefalotórax das fêmeas ser maior que o dos machos. Bueno et al. (2000) encontraram peso médio máximo de machos inferiores $\left(\mathrm{P}_{\mathrm{t}}=2,48\left[1-\mathrm{e}^{-0,0041 \mathrm{t} t+}\right.\right.$ $\left.{ }_{39,13)}^{3,04}\right)$ e em fêmeas foram superiores $\left(\mathrm{P}_{t}=3,38\left[1-\mathrm{e}^{-0,0033(\mathrm{t}+}\right.\right.$ $50,38)]^{3,05}$ ) comparados com os valores obtidos com a análise do deslocamento modal da espécie aqui estudada.

Para análise da hipótese de existência de heteroquelia foram analisadas as medidas de 134 animais, sendo 90 machos e 44 fêmeas. Pela análise de covariância foram comparadas as regressões do comprimento da quela direita com o comprimento da quela esquerda, para machos e para fêmeas. A hipótese de homogeneidade das variâncias residuais $\left(\mathrm{F}_{\mathrm{c}}=1,31\right.$ $\left.<\mathrm{F}_{0,05, \mathrm{gl} \mathrm{88,42}}\right)$, de igualdade entre as declividades $\left(\mathrm{F}_{\mathrm{c}}=0,005<\right.$ $\left.\mathrm{F}_{0,05, \mathrm{gl} 1,130}\right)$ e de igualdade nas elevações da reta $\left(\mathrm{F}_{\mathrm{c}}=0,7<\right.$ $\left.\mathrm{F}_{0,05, \mathrm{gl} 1,131}\right)$ foram aceitas, não sendo constatadas diferenças significativas com intervalo de confiança de $95 \%$.

Este resultado difere do encontrado para as demais espécies pesquisadas. BAHAMONDE \& LóPEZ (1961) destacam que em 
machos de A. laevis laevis os quelípodos são mais desenvolvidos e desiguais. López (1965), constatou em A. odebrecthii paulensis que em ambos os sexos os quelíopodos são desiguais, sendo que $72 \%$ dos machos e $82,5 \%$ das fêmeas apresenta o quelípodo esquerdo mais desenvolvido e em $A$. perobae, tal proporção foi $78,58 \%$ e 76,38\%, respectivamente (Rodrigues \& Hebling 1978). BUENo et al. (2000) registram, em A. platensis 36,5\% dos machos com quela esquerda maior enquanto $51,7 \%$ das fêmeas apresentaram o tamanho das quelas iguais.

\section{REFERÊNCIAS BIBLIOGRÁFICAS}

AlLen, R.L. 1976. Method for comparing fish growth curves. New Zeland Journal of Marine and Freshwater Research, Wellington, 10 (Supl. 4): 687-692.

ArenAS, R.L. 1976. La cordillera de la costa como refugio de la fauna dulcícola preglacial. Archivos de Biologia y Medicina Experimentales, Santiago, 10: 1-40.

BAhamonde, N. \& M.T. López. 1961. Estudios biológicos en la populación de Aegla laevis laevis (Latreille) de el Monte (Crustacea, Decapoda, Anomura). Investigaciones Zoológicas Chilenas, Santiago, 7: 19-58.

BertalanfFy, L. 1938. A quantitative theory of organic growth. Human Biology, Deroit, 10 (2): 181-213.

Bond-Buckup, G. \& L. Buckup. 1994. A família Aeglidae (Crustacea, Decapoda, Anomura). Archivos de Zoologia, São Paulo, 2 (Supl. 4): 159-346.

Bueno, A.A.P. \& G. BOND-BucKuP. 2000. Dinâmica populacional de Aegla platensis Schmitt (Crustacea, Decapoda, Aeglidae). Revista Brasileira de Zoologia, Curitiba, 17 (Supl. 1): 4349.

Bueno, A.A.P.; G. Bond-Buckup \& L. Buckup. 2000. Crescimento de Aegla platensis Schmitt em ambiente natural (Crustacea, Decapoda, Aeglidae). Revista Brasileira de Zoologia, Curitiba, 17 (Supl. 1): 51-60.

Fabens, A.J. 1965. Properties and fitting of the von Bertalanffy growth curve. Growth, Lakeland, (29): 265-289.

Hartnoll, R.G. 1982. Growth, p.111-196. In: D.E. Bliss (Ed.). The Biology of Crustacea. New York, Academic Press, Vol.2, 440p.

. 1985. Growth, sexual maturity and reprodutive output, p.101-128. In: A.M. WENNER (Ed.). Crustacean Issues: Factors in Adult Growth. Rotterdam, A.A. Balkema, vol. 3, 362 .

JARA, C. 1992. Aegla expansa, new species (Crustacea: Decapoda: Anomura: Aeglidae), from the lower Bio-bio River Basin,
Concépcion, Chile. Gayana Zoologia, Concepcion, 56 (12): $49-57$.

. 1994. Aegla pewenchae, a new species of central Chilean freshwater decapod (Crustacea: Anomura: Aeglidae). Proceedings of the Biological Society of Washington, Washington, D.C., 107 (Supl. 2): 325-339.

López, M.T. 1965. Estudios biologicos en Aegla odebrechtti paulensis, Schmitt (Crustacea, Decapoda, Anomura). Boletin de Zoologia, Faculdade de Filosofia, Ciências e Letras, São Paulo, 25: 301-314.

Magni, S.T. \& V. Py-Daniel. 1989. Aegla platensis Schmitt, 1942 (Decapoda, Anomura) um predador de imaturos de Simuliidae (Diptera, Culicomorpha). Revista de Saúde Pública, São Paulo, 23 (Supl. 3): 258-259.

Passano, L.M. 1960. Molting and its control, p.473-536. In: H.W. Tадвот (Ed.). The Physiology of Crustacea: metabolim and growth. New York, Academic Press, vol. 1, 670p.

RICKER, W.E. 1975. Computation and interpretation of biological statistics of fish populations. Bulletin of the Fisheries Research Board of Canada, Ottawa, 191: 1-382.

Rodrigues, W. \& N.J. Hebling. 1978. Estudos biológicos em Aegla perobae Hebling \& Rodrigues, 1977 (Decapoda, Anomura). Revista Brasileira de Biologia, Rio de Janeiro, 38 (Supl. 2): 383-390.

SANTos, E.P. 1978. Dinâmica de populacões aplicada à pesca e piscicultura. São Paulo, HUCITEC/EDUSP, 129p.

Snedecor, G.W. \& W.G. Cochran. 1967. Statistical Methods. Ames, Iowa State University Press, $6^{\mathrm{a}}$ ed., 593p.

Swiech-Ayoub, B.P. \& S. Masunari. 2001a. Flutuações temporal e espacial de abundância e composição de tamanho de Aegla castro Schmitt (Crustacea, Anomura, Aeglidae) no Buraco do Padre, Ponta Grossa, Paraná, Brasil. Revista Brasileira de Zoologia, Curitiba, 18 (Supl. 3): 1003-1017.

Swiech-Ayoub, B.P. \& S. Masunari. 2001b. Biologia reprodutiva de Aegla castro Schmitt (Crustacea, Anomura, Aeglidae) no Buraco do Padre, Ponta Grossa, Paraná, Brasil. Revista Brasileira de Zoologia, Curitiba, 18 (Supl. 3): 1019-1030.

Vaz-Ferreira, R.; R. Gary \& M. Vaz-Ferreira. 1945. Notas biométricas sobre los crustáceos decapodos del gênero Aegla Leach, I. La variación de algunas magnitudes em Aegla uruguayana Schmitt. Communicaciones Zoologicas del Museo de História natural de Montevideo, Montevideo, 1 (Supl. 24): 1-6.

WALFORD, L.A. 1946. A new graphic method of describing the growth of animals. Biological Bulletin, Woods Hole, 90 (2): 141-147.

Recebido em 10.X.2002; aceito em 28.IV.2003. 